\title{
EDUCACIÓN PARA LA SALUD SEXUAL EN LA FORMACIÓN DE PROFESORES EN ARGENTINA
}

\section{Sexual Health Education in teacher training in Argentina}

\author{
Elsa Meinardi ${ }^{1}$ \\ Andrea Revel Chion ${ }^{2}$ \\ Elina Godoy ${ }^{3}$ \\ María Iglesias ${ }^{4}$ \\ Inés Rodríguez Vida ${ }^{5}$ \\ María Victoria Plaza ${ }^{6}$ \\ Leonor Bonan ${ }^{7}$
}

\begin{abstract}
Resumen: En este trabajo formulamos apreciaciones acerca del estado de situación de la formación docente en educación sexual en Argentina y proponemos algunos interrogantes a fin de pensar cómo se configura este problema de investigación. Al mismo tiempo, presentamos algunos datos preliminares sobre un trabajo de indagación realizado por profesoras de enseñanza media con estudiantes de dos grupos etarios diferenciados: adolescentes de 14 a 16 años y estudiantes adultos que retoman sus estudios después de varios años de alejamiento de la educación formal. Nuestro objetivo fue relevar algunas concepciones de los estudiantes, de modo que éstas pudieran servir para orientar mejor la planificación de clases sobre Educación para la Salud Sexual.
\end{abstract}

Palabras clave: Educación sexual. Formación de profesores. Salud reproductiva.

\begin{abstract}
In this work, we made assessments about the status of teacher training in sex education in Argentina and we propose some research problems. At the same time, we present some preliminary data from high school students in two distinct age groups: teenagers 14 to 16 years of age and adults who resumed their studies after several years out of formal education. Our objective was to explore some conceptions of students, so they could serve to guide for the planning classes on Sexual Health.
\end{abstract}

Keywords: Sexual education. Teacher training. Reproductive health.

\footnotetext{
1-7 Investigadores y docentes del Grupo de Investigación en Didáctica de la Biología, Centro de Formación e Investigación en Enseñanza de las Ciencias, Facultad de Ciencias Exactas y Naturales, Universidad de Buenos Aires, Argentina. <emeinardi@fibertel.com.ar>
}

\footnotetext{
${ }^{1}$ Agustín Álvarez 1232, Vicente López

(1638) Provincia de Buenos Aires, Argentina
} 


\section{Introducción}

La investigación sobre la formación del profesorado en Educación para la Salud Sexual es un campo que cuenta con escasos antecedentes en Argentina. Es así que en nuestra tarea como formadores de formadores nos encontramos con muy pocas orientaciones, tanto curriculares como didácticas, para desarrollar acciones que brinden a nuestros estudiantes futuros profesores de biología- herramientas para diseñar e implementar estrategias efectivas en el aula.

Esta falta de investigación, y la consecuente carencia de orientaciones y de reflexión sobre el tema, podría llevar a que los formadores de formadores tampoco estemos capacitados para trabajar en Educación para la Salud Sexual.

En atención a esta situación - en el marco de un programa de investigación didáctica, desde hace varios años el propósito de nuestro grupo es desarrollar acciones de formación de profesores en estrecha relación con la intervención en las escuelas. De este modo pretendemos contribuir a delinear un posible currículo de formación docente, al mismo tiempo que diseñar e implementar prácticas escolares fundamentadas.

En este trabajo presentamos un relevamiento de diferentes aspectos relacionados con la Educación para la Salud Sexual. En primer lugar, nos centramos en el desarrollo de un marco conceptual que nos permite caracterizar la Educación para la Salud Sexual en relación con el ambiente y la salud. Luego, exponemos diferentes relevamientos sobre la educación sexual en el ámbito escolar: el vínculo existente entre la formación docente y las prácticas de la enseñanza, la presencia de contenidos relacionados con la educación sexual en el currículo de la formación docente y en el currículo escolar. Por último, presentamos un relevamiento de las concepciones de los estudiantes (adolescentes y adultos) sobre la anticoncepción realizado a través del diseño, la elaboración y la aplicación de una encuesta, cuyos resultados se comentan sobre el final del apartado. El trabajo culmina con una serie de reflexiones que decantan de las acciones antes descriptas.

\section{Contexto de emergencia de la Educación para la Salud Sexual}

Para apreciar la envergadura social del tema que nos convoca nos parece importante repasar algunas cifras en relación con la población de Argentina (INDEC, 2001):

- Población total del país: 36 millones de personas.

- Población de la Ciudad Autónoma de Buenos Aires8: 3 millones.

- Población de la Provincia de Buenos Aires': 14 millones.

- Población con Necesidades Básicas Insatisfechas ${ }^{10}$ (total del país): 6 millones (17 \%).

${ }^{8}$ La Ciudad de Buenos Aires es la de mayor densidad poblacional del país.

${ }^{9}$ La provincia de Buenos Aires cuenta con la mayor cantidad de habitantes del país. 
En la Argentina:

- La salud sexual compromete el derecho a la salud de 21 millones de personas: mujeres en edad fértil, niños y niñas y adolescentes.

- Se practican unos 500.000 abortos al año (el aborto es ilegal).

- El aborto causa el $40 \%$ de las muertes en mujeres de 20 a 29 años y el $31 \%$ de las muertes en menores de 20 años.

- Los riesgos de mortalidad infantil se duplican si se trata de madres adolescentes (45\% en madres menores de 15 años).

En la provincia de Buenos Aires:

- Casi 35 mil mujeres por año (unas 95 por día) llegan a los hospitales públicos por abortos y complicaciones post aborto.

- Tres mujeres por mes mueren por este tipo de prácticas.

- El registro no oficial indica que todos los días alrededor de 350 mujeres deciden interrumpir su embarazo.

- La maternidad adolescente representa el 25\% de los nacimientos.

Esta información contribuye a poner de manifiesto la urgencia de generar mecanismos de intervención social eficaces, que colaboren a modificar esta situación. Y dentro de estos mecanismos la educación formal debe ocupar un lugar destacado.

\section{Algunas consideraciones acerca de los marcos teóricos de la Educación para la Salud Sexual}

\section{Educación Ambiental y Educación para la Salud}

El concepto de salud ha ido cambiando en los últimos tiempos y se ha abandonado la idea de salud como "ausencia de enfermedad". Desde hace unos años la OMS (1985 apud GAVIDIA e RODES, 1999, p. 89) define la salud como "la capacidad de desarrollar el propio potencial personal y de responder de forma positiva a los retos del ambiente".

Ahora bien, ¿cómo concebimos actualmente el ambiente? Más allá de las diferencias que se puedan establecer, hay un acuerdo básico en considerarlo algo más que un conjunto de elementos físicos y químicos que interactúan con los componentes biológicos; los aspectos sociales, tales como la pobreza, las carencias educativas, la discriminación por cuestiones de género y las prácticas aceptadas y rechazadas en un determinado espacio geográfico y tiempo histórico son también componentes del ambiente (MEINARDI, ADURIZ-BRAVO e RIVEL-CHION, 2002).

\footnotetext{
${ }^{10}$ Los hogares con necesidades básicas insatisfechas presentan al menos uno de los siguientes indicadores de privación: hacinamiento (hogares que tienen más de tres personas por habitación), vivienda precaria (hogares que habitan en una vivienda de tipo inconveniente, precaria etc.), condiciones sanitarias (hogares que no tienen ningún tipo de retrete), asistencia escolar (hogares que tienen algún niño en edad escolar que no asista a la escuela), capacidad de subsistencia (hogares que tienen cuatro o más personas por miembro ocupado y, además, cuyo jefe tiene baja educación).
} 
Meinardi, E. et al.

En este marco, considerando el impacto del ambiente sobre la salud, la Educación Ambiental no puede dejar de lado la Educación para la Salud (incluyendo en ella la Educación Sexual). Tanto en la Educación Ambiental como en la Educación para la Salud el énfasis está puesto en lograr un cambio de actitud en las personas; además tienen en común el peso que asumen en ellas las problemáticas de género. Así como no se puede pensar en una educación para el desarrollo y la sostenibilidad del ambiente sin tener en cuenta el papel que desempeñan las asignaciones de género en cada cultura, no se puede imaginar una educación para la salud que no las considere.

\section{Educación para la Salud y Educación Sexual}

La Educación para la Salud se sustentó durante muchos años en transmitir información sobre los fundamentos científicos de los problemas de salud y sobre el peligro de ciertas conductas. Bajo esta perspectiva, se espera que el acceso a la información transforme de inmediato las prácticas sexuales juveniles, instaurando una conducta de auto-protección que eliminaría posibles riesgos (BRANDÃO e HEILBORN, 2006).

Más recientemente, numerosos trabajos (véase por ejemplo AROMI et al., 2002) han mostrado que el conocimiento no alcanza para modificar las conductas. Así, el enfoque de transmisión de información dio paso a la promoción de actitudes y motivaciones que faciliten la modificación de conductas.

Actualmente se ha producido un nuevo cambio que intenta evitar los modelos sanitarios manipuladores; se admite la necesidad de que sea el propio individuo quien voluntariamente adopte determinadas decisiones respecto a sus formas de vivir, que sea él mismo quien decida si admite ciertos riegos o los rechaza. De esta forma, las estrategias de enseñanza se dirigen a desarrollar las capacidades para la toma de decisiones suficientemente informadas. Para evitar que la educación sanitaria se centre exclusivamente en los aspectos conductuales, se debe introducir la responsabilidad que en la salud poseen las condiciones y los estilos de vida.

Esta dimensión cultural es importante porque no estamos sanos por lo que sabemos sino por lo que hacemos (GAVIDIA e RODES, 1996). De acuerdo con ello, algunos autores mencionan que las propuestas dirigidas a los jóvenes llevan implícito el mensaje de que la salud es algo que "deben ahorrar ahora para tener después" (GAVIDIA e RODES, 1999, p. 92), pero esto no parece acorde con la estructura psicológica de los adolescentes; la cultura adolescente postula el aquí y abora como modo de tránsito en la vida.

Pensar en una Educación para la Salud Sexual efectiva viene condicionado por todos estos factores revisados, lo que plantea desafíos importantes a la hora de llevarla a la escuela. El aula es el espacio de síntesis en el que se concretan un gran número de variables, algunas de las cuales hemos mencionado en los párrafos anteriores. A estas debemos sumarle, entre otras, las relacionadas con la formación docente, la práctica profesional y el currículo escolar, además de las inherentes a las concepciones del profesorado y de los propios estudiantes, tanto en relación con la sexualidad como con la educación sexual. Mencionaremos a continuación algunos aspectos vinculados con ellas. 
Educación para la Salud Sexual en la formación...

\section{Educación para la Salud Sexual y escuela}

\section{La formación docente y las prácticas de enseñanza}

Una encuesta realizada en la Argentina por el Consorcio Nacional de Derechos Reproductivos y Sexuales (CONDERS, 2003) a 260 personas (aproximadamente dez encuestas por provincia en todo el país) mostró que, para los jóvenes, el compromiso y la capacitación de los profesores es un factor muy importante que favorece la Educación para la Salud Sexual es, mientras que un profesorado no capacitado y con miedo la dificulta. Consultados acerca de si consideran necesaria la Educación para la Salud Sexual, el 98,8\% de los jóvenes respondió afirmativamente. Otra encuesta realizada a aproximadamente 100 profesores, dio como resultado que casi al $40 \%$ le había resultado embarazoso contestar las dudas que los adolescentes planteaban sobre el tema (CAAMAÑO CANO, 2003).

En instancias de trabajo con los profesores y profesoras hemos llegado a recoger la opinión de que la incomodidad que sienten ante preguntas de los estudiantes que van más allá de lo esperado (¿debo responder preguntas de opinión?, ¿hasta dónde debo responder?, etcétera) obedece, en muchos casos, a la falta de espacios compartidos de reflexión y a la carencia de orientaciones didácticas, es decir, a la ausencia de formación acerca de cómo tratar estos temas en el aula. El profesorado, entonces, "hace lo que puede", enfrentándose a estas situaciones desde el severamente criticado "sentido común", al mismo tiempo que las cuestiones de género ni siquiera son contempladas como temas posibles de ser abordados por los profesores y profesoras de biología.

Como señalan Brêtas y Silva (2005) en este mismo sentido, la escuela, por su importancia en el campo de la socialización del escolar y del adolescente, sería un vehículo muy importante para la educación sexual, pero debido a variables como la falta de preparación de los profesores para la discusión del tema, la ideología por la cual para dominar una situación usan mecanismos de control como la represión o la biologización de la sexualidad, con la connivencia de las ciencias médicas, sólo se logra vincular el ejercicio de la sexualidad con la práctica de las funciones reproductoras.

Barcelos et al., en un trabajo de 1996, señalaban que los educadores, en su mayoría, "tratan el tema apenas en sus aspectos biológicos, o como máximo, invitan a un expositor médico para eso" (BARCELOS, ZAIAD e SANTOS, 1996, p. 151). Esta afirmación, que describía la situación escolar de Brasil hace más de diez años, sigue siendo aún hoy completamente válida para Argentina. En las escuelas se apela a un "agente externo" para el tratamiento de estos temas. Es muy frecuente que se convoque a un profesional de la salud, médico o ginecólogo, quien ofrece una charla a los estudiantes muchas veces con la presencia de adultos, padres, madres y autoridades escolares. De esta forma se cumple con la formalidad de "dar educación sexual", en un contexto que la institución considera seguro. Este modelo conceptualmente perimido de educación para la salud sigue aún muy vigente posiblemente porque tranquiliza, ya que no se producen preguntas conflictivas de los estudiantes y la situación está bajo el control de los adultos. De hecho, se lo considera perimido justamente porque ésta es la única función que cumple: dar información.

En un estudio reciente también realizado en Brasil, Silva y Carvalho (2005) mencionan que la falta de preparación del profesorado para trabajar con las cuestiones de sexualidad en la escuela puede tener origen en la educación familiar antisexual y opresora que ellos recibie- 
ron y, también, en su formación académica inicial, en la que hay poca discusión sobre esta temática.

Ramos (2007), al investigar las concepciones de un grupo de docentes de una escuela media respecto de la sexualidad y de la educación sexual, muestra claramente cómo las explicaciones del sentido común en temas de sexualidad impregnan continuamente sus prácticas. En esta línea, cada vez más se reconoce el impacto que producen las concepciones implícitas de los docentes sobre su desempeño profesional. Es así que, según Bernardi (1985), la manera predominante del modelo de educación sexual es aquella en la que el educador intenta eliminar su propia sexualidad, y anhela que se sofoque también la sexualidad de los educandos.

Es de esperar que la toma de conciencia de los profesores del tipo de explicaciones que emplean en sus clases brinde elementos para que ellos puedan enriquecer, analizar críticamente y mejorar sus estrategias de enseñanza. Sin embargo, este camino no es sencillo dado que la producción didáctica sobre la formación de los formadores de formadores es prácticamente inexistente, lo cual agrava aún más la situación. Para capacitar a los profesores y profesoras en estos temas, y capacitarnos nosotros -los encargados de su formación-, el enfoque que proponemos se sustenta en una concepción del conocimiento científico como un proceso social, producto de las vinculaciones interactivas entre unidades productoras de conocimiento, usuarios y profesorado.

Se trata de conducir la educación hacia la construcción de la sexualidad entendida como una experiencia histórica y personal, una construcción que no se agota en la información sobre sexualidad sino que incluye además la formación de actitudes ${ }^{11}$ hacia lo sexual (SÁNCHEZ e SANTOS, 1986). Mandú y Corrêa (2000) refieren que la sexualidad articula procesos biológicos y socioculturales, que sin embargo tienen una expresión singular en cada sujeto; es un proceso subjetivo, mediado a través del cuerpo, de la experiencia, de los cambios, de las búsquedas, de las proyecciones construidas en la vida. La sexualidad es un componente humano que se define a partir de la compleja interrelación entre biología, subjetividad y condiciones existenciales concretas. Esta relación entre el cuerpo biológico, la subjetividad y las influencias socioculturales, es la que da especificidad al ejercicio de la sexualidad de los diferentes grupos (BRÊTAS e SILVA, 2005).

Considerando estas perspectivas, podemos afirmar que la Educación para la Salud Sexual es un contenido hasta ahora excluido de la educación formal en Argentina. Como menciona Santos (2006) al referirse al sistema educativo en nuestro país, aún no se ha logrado que la escuela aborde, como parte de su currículo, el análisis de los distintos aspectos que se encuentran articulados en la compleja sexualidad humana.

\section{Los contenidos de la formación docente}

Hemos llevado adelante una búsqueda con el fin de determinar la presencia de contenidos de Educación para la Salud Sexual (desde una perspectiva no meramente biologicista,

\footnotetext{
${ }^{11}$ Entendiendo por actitud una organización relativamente duradera de creencias que predispone a la persona a responder de determinada manera frente a una situación (ROKEACH, 1974). 
sino como la que presentamos más arriba) en los currículos de la formación docente inicial y hallamos que, muchas veces, dichos currículos son igualmente carentes que los de la educación media (MEINARDI, 2007, 2005). Si bien existen numerosos ámbitos de educación no formal sobre la temática, no se ha logrado concretar una selección de contenidos, y mucho menos una secuenciación, para integrar un currículo nacional de formación docente.

Por otro lado, es cada vez más claro que en relación con la formación docente no sólo son relevantes los contenidos disciplinares que puedan integrar el currículo sino que, como señalan Silva y Carvalho (2005, p. 74), el desenvolvimiento profesional de los profesores va a depender fuertemente del "conocimiento pedagógico del contenido", el cual va más allá del conocimiento de la disciplina en sí, hacia una dimensión del conocimiento de la disciplina para la enseñanza (SHULMAN, 1986). El conocimiento profesional deseable es un conocimiento epistemológicamente diferenciado, resultado de la elaboración e integración de diferentes saberes; es un conocimiento que contiene determinadas actitudes y valores encaminados a la transformación escolar y profesional.

\section{La Educación Sexual en el currículo escolar}

En relación con la inserción de los contenidos de la Educación Sexual en el currículo de la escuela media, es interesante notar que hasta hace unas cuatro décadas ni siquiera la mirada más biologicista, centrada en la enseñanza del "aparato reproductor", formaba parte de los contenidos curriculares de la escuela media de Argentina. Actualmente todavía se discute en algunos ámbitos si conviene o se debe enseñar anticoncepción; el debate profundo acerca de la Educación para la Salud Sexual como un contenido que va más allá de la salud reproductiva es todavía una deuda pendiente en la educación formal de nuestro país.

Los conocimientos sobre el aparato reproductor son relevantes para la alfabetización científica, ya que permiten a los ciudadanos ser conscientes de las problemáticas del mundo que los rodea de manera tal que queden habilitados para la toma de decisiones sobre su propia vida. Sin embargo, la salud sexual es una construcción que no solo está vinculada con ser capaz de evitar enfermedades de transmisión sexual y embarazos no planificados (DENTE e ROGALSKI, 1999) o con el mero reconocimiento de la anatomía de un sistema; la vida sexual es muy compleja y va mucho más allá de las funciones biológicas de nuestro aparato reproductor. Como menciona Gavidia (2004), no por tratar en el aula la anatomía humana hacemos Educación para la Salud Sexual.

Al mismo tiempo, tradicionalmente las secuencias de enseñanza más frecuentemente halladas en las clases de biología se corresponden con una propuesta curricular que involucra, primero el tratamiento de las estructuras del "aparato reproductor", luego las funciones vinculadas con la reproducción (ciclo menstrual, fecundación etc.) y posteriormente, en algunos casos, los métodos anticonceptivos. Nuestra experiencia como docentes, tanto en escuelas secundarias como en la formación de profesores y profesoras en el ámbito de la universidad, nos indica que el tratamiento del sistema reproductor se limita casi exclusivamente a la descripción de los órganos que lo conforman, a su función reproductiva y a las infecciones de transmisión sexual (antes llamadas ETS-enfermedades de transmisión sexual).

La equivalencia entre sexualidad y reproducción deja afuera del análisis aspectos muy relevantes para los jóvenes, como aquellos vinculados con el placer y la subjetividad, es decir, a una verdadera concepción de la vida sexual. Desde la perspectiva tradicional, el sistema es 
"reproductor", mientras que sus enfermedades son "sexuales" y los métodos anticonceptivos forman parte de la "planificación familiar".

Como menciona Scholer (2002), la respuesta segura para ocuparse del sexo y de la sexualidad es reducir el tema a la anatomía del sistema reproductor y al ciclo hormonal. Sin embargo, la "caja fuerte" no resuelve las necesidades de los estudiantes. Se ha visto que no alcanza con enseñar sistema reproductor ( $y$ aún salud reproductiva) para promover en los adolescentes conductas fundamentadas sobre la salud sexual. Además, es necesario que los profesores y profesoras de biología que deciden enseñar educación sexual estén capacitados para hacerlo.

En relación con la selección de los contenidos de un currículo escolar de Educación para la Salud Sexual, pensamos que estos podrían organizarse en cinco ejes fundamentales:

- Representaciones y valores de la salud reproductiva

- Modelos sexuales y de género

- Anticoncepción y aborto

- Derechos sexuales y reproductivos

- Prevención de infecciones transmitidas por vía sexual

Para generar una reflexión de los profesores acerca de la elección de contenidos, además de la discusión sobre qué conocimientos deben ser transferidos, en qué casos y, si fuera necesario, a través de qué instancias, es necesario comenzar revisando el para qué de dichos conocimientos, los objetivos que se persiguen a través de ellos.

Ceruti Baso (1995) propone una vasta lista sobre los objetivos que debería perseguir la formación de los estudiantes en esta temática, de la cual consideramos prioritarios:

- Lograr el desarrollo de un pensamiento crítico que posibilite la adopción de actitudes positivas hacia la sexualidad.

- Favorecer el proceso a través del cual es posible reconocerse, identificarse y aceptarse como ser sexual.

- Favorecer el desarrollo de roles sexuales en el marco de una dialéctica de valores basada en los derechos humanos, que propicie relaciones de respeto y equidad entre las personas.

- Favorecer un mayor conocimiento y vínculo con el propio cuerpo, como elemento de autoestima y de sustento del autocuidado de la salud.

- Favorecer la adopción de conductas sexuales conscientes, placenteras y responsables hacia uno mismo y los demás.

\section{Educación sexual en la educación de adultos}

Dado que algunos de los participantes del grupo de trabajo y muchos de nuestros estudiantes de profesorado se desempeñan como docentes en la educación secundaria de adultos, consideramos interesante averiguar si había investigaciones acerca de la educación sexual en la educación media para este grupo etario en particular. En Argentina la población de adultos que cursan la enseñanza secundaria se estima en unas 400 mil personas mayores de 18 años, un tercio de los cuales tiene más de 25 años. Cross (1981) mencionaba que la base teórica de la investigación de la educación de adultos es prácticamente "inexistente". Nuestra propia indagación acerca del estado actual de la investigación sobre la Educación para la Salud Sexual en la enseñanza media para adultos arroja los mismos resultados, 27 años después. 
Educación para la Salud Sexual en la formación...

A la complejidad de la educación para adultos debemos sumarle la de la educación formal en temas de Salud Sexual. ¿En qué se parece y en qué se diferencia de la educación de jóvenes? ¿Necesitan los adultos ser educados en temas de sexualidad? Creemos que sí, pero aún estamos investigando la naturaleza idiosincrásica de esta educación. Por otra parte, nos preguntamos si las concepciones de ambos grupos son similares o se diferencian notablemente.

\section{Las concepciones de los estudiantes sobre la salud sexual}

Un relevamiento realizado en un servicio de salud de un hospital público de la Ciudad de Buenos Aires (SANTOS, 2003) muestra que algunos jóvenes consideran que si la mujer toma pastillas anticonceptivas no necesitan usar preservativos durante las relaciones sexuales; estos y otros trabajos indican que muchas veces no hay una distinción clara entre las funciones anticonceptivas y la prevención de infecciones de transmisión sexual.

En un trabajo anterior (REVEL CHION, BONAN e MEINARDI, 2005) comentamos que al interrogar a los adolescentes acerca de las formas de evitar el embarazo y prevenir el contagio del VIH, algunos mencionaban: "es poco viril usar profilácticos" o "en el momento de la relación sexual la decisión la toma el varón".

Más recientemente, algunas de las participantes del grupo de trabajo que dan clase en escuelas secundarias entrevistaron a sus estudiantes para conocer las concepciones pre-instruccionales respecto de algunos ítems, con el fin de orientar la planificación de sus clases. Este trabajo es la primera etapa del proyecto que tiene como principales características la realización de una actividad exploratoria, sistemática y cooperativa, llevada a cabo por los profesores que, al mismo tiempo, participan de un proyecto de investigación (DAPÍA CONDE, MEMBIELA e CID, 1996). Luego de esta etapa se llevó a cabo la enseñanza de la temática.

\section{Metodología}

Para la toma de datos fue utilizado un cuestionario escrito, con preguntas de respuesta cerrada, aplicado individualmente y en el ámbito de la escuela. Los ítems fueron elaborados en base a una serie de mitos en relación con la sexualidad que circulan socialmente, en especial, en las clases populares (MARGULIS, 2003). En la Tabla 1 se muestran las preguntas que formaron parte del cuestionario. Los entrevistados respondieron de manera anónima, indicando únicamente el sexo y la edad. Las entrevistadoras, integrantes del grupo de investigación, son al mismo tiempo docentes de las materias Biología y Educación para la salud de los cursos a los que pertenecen las y los alumnos entrevistados.

Conformación de los grupos de entrevistados:

- Grupo A: formado por 31 estudiantes adultos, mayores de 18 años, alumnos y alumnas de dos cursos de segundo año de dos escuelas para adultos. La mayoría de los estudiantes pertenece a niveles socioeconómicos bajos y retoma sus estudios después de varios años de alejamiento de la educación formal.

- Grupo B: formado por 72 estudiantes de 14 a 16 años, mujeres y varones de dos cursos de cuarto año de la misma escuela. La mayoría de los estudiantes pertenece a niveles socioeconómicos medios y altos. 
Meinardi, E. et al.

El cuestionario fue pasado en un momento del año en el cual todavía no se habían tratado los temas relacionados con la reproducción, la anticoncepción y el ciclo hormonal, por eso lo denominamos pre-instruccional. En esta indagación nos centramos en consultar a los entrevistados acerca de posibles situaciones que pudieran o no conducir a un embarazo. Para la elaboración de los ítems utilizamos fuentes que informan acerca de mitos de trascendencia social sobre las situaciones que conducen a embarazos (SANTOS, 2006; MARGULIS, 2003; PEREIRO NÚÑEZ e PIZARRO SÁNCHEZ, 1997; LÓPEZ GARCÍA, 1995).

\section{Resultados}

En la Tabla 1 se muestra la proporción de aciertos en las respuestas; en los casos en que no se indica, los porcentajes se reparten de manera similar entre quienes dan respuestas erróneas y quienes no saben o no contestan. Los estudiantes debían responder si las situaciones relatadas en la tabla podrían o no conducir a embarazos.

Tabla 1. Resultados de la encuesta a dos grupos de estudiantes.

\begin{tabular}{|c|c|c|c|c|}
\hline \multirow{3}{*}{$\begin{array}{l}\text { ¿Las siguientes situaciones pueden } \\
\text { conducir a embarazos? }\end{array}$} & \multirow{2}{*}{\multicolumn{2}{|c|}{$\begin{array}{c}\text { \% Aciertos } \\
\text { Grupo A (31 adultos } \\
\text { mayores de } 18 \text { años) }\end{array}$}} & \multirow{2}{*}{\multicolumn{2}{|c|}{$\begin{array}{c}\% \text { Aciertos } \\
\text { Grupo B (72 jóvenes } \\
\text { de } 14 \text { a } 16 \text { años })\end{array}$}} \\
\hline & & & & \\
\hline & 19 Mujeres & 12 Varones & 36 Mujeres & 36 Varones \\
\hline No cuidarse en la primera relación sexual & 68 & 91 & 94 & 86 \\
\hline Relaciones sexuales durante la lactancia & 84 & 75 & 43 & 43 \\
\hline $\begin{array}{l}\text { Relaciones sexuales durante la } \\
\text { menstruación }\end{array}$ & $\begin{array}{c}26(26 \% \\
\text { desaciertos y } 47 \\
\% \text { no sabe })\end{array}$ & $\begin{array}{c}25(75 \% \\
\text { desaciertos) }\end{array}$ & 31 & 38 \\
\hline $\begin{array}{l}\text { *Orinar antes y después de la relación } \\
\text { sexual }\end{array}$ & 50 & 50 & - & - \\
\hline $\begin{array}{l}\text { Lavarse la mujer después de la relación } \\
\text { sexual }\end{array}$ & 36 & 58 & 47 & 62 \\
\hline $\begin{array}{l}\text { Compartir la pileta de natación con } \\
\text { hombres }\end{array}$ & 42 (42\% no sabe) & 66 & 58 & 42 \\
\hline $\begin{array}{l}\text { Ponerse de pie durante o después de la } \\
\text { relación sexual }\end{array}$ & 42 (53\% no sabe) & 23 & 51 & 55 \\
\hline Retirar el pene antes de la eyaculación & 63 & 42 & 51 & 33 \\
\hline $\begin{array}{l}\text { Penetración sexual sin preservativo y } \\
\text { colocación antes de la eyaculación }\end{array}$ & 68 & 67 & 44 & 40 \\
\hline
\end{tabular}

* Esta pregunta no estaba presente en el cuestionario dado al Grupo B. 
En la elaboración del cuestionario no tuvimos en cuenta la probabilidad de la ocurrencia del embarazo ante cada una de las situaciones presentadas, pues sabemos que este es un concepto de muy alto grado de abstracción que se constituye en un obstáculo de aprendizaje en sí mismo. Excepto en el caso de "compartir la pileta de natación con hombres", evaluamos como respuestas correctas aquellas que indicaban que las situaciones planteadas podían conducir a embarazos.

Se desprende de la tabla que un porcentaje bastante alto (32\%) de mujeres adultas considera, erróneamente, que no hay posibilidades de embarazo durante la primera relación sexual. En un trabajo llevado a cabo en Brasil por Teixeira et al. (2006) se encontró que sobre 4019 jóvenes de 18 a 24 años de ambos sexos, un 30 \% había mantenido su primera relación sexual sin hacer uso de ningún método de protección, y de éstos un $13 \%$ de mujeres y 9,6\% de varones consideraba que en esa ocasión no podía ocurrir el embarazo.

Respecto de la posibilidad de embarazo durante la lactancia, la mayoría de las respuestas del grupo de adultos son correctas; lo contrario ocurre con las respuestas de los jóvenes. Esto puede estar relacionado con que muchas veces la lactancia materna es considerada un método anticonceptivo en sí mismo, con desconocimiento de que sólo en determinadas circunstancias esto ocurre (lactancia exclusiva, con poco intervalo entre tomas, hasta los seis meses de edad etc...).

Acerca de si mantener relaciones sexuales durante la menstruación puede conducir a un embarazo (consideramos que sí, independientemente de la probabilidad de ocurrencia ya que, aunque baja, no es nula), los porcentajes de respuestas incorrectas son llamativamente altos. Un $26 \%$ de mujeres adultas responde que es posible el embarazo, un $26 \%$ que no es posible y casi la mitad no sabe; el $75 \%$ de los varones de esta edad piensa que no hay posibilidad de embarazo durante la menstruación. Entre los jóvenes, alrededor de un 60-70\% responde incorrectamente o no sabe. ¿Lo considerarán también una forma de método anticonceptivo mantener relaciones durante la menstruación? ¿ $\mathrm{O}$ no les preocupa el tema porque la mayoría no mantiene relaciones durante ese período? No lo sabemos.

Sólo un $42 \%$ de las mujeres adultas y los varones jóvenes no considera (correctamente) que compartir una pileta de natación con hombres es una situación de riesgo de embarazo.

"No cuidarse durante la primera relación" fue la situación que muestra el mayor porcentaje de respuestas correctas, aunque las mujeres adultas (y de niveles socioeconómicos bajos) produjeron el mayor número de respuestas erradas; "relaciones sexuales durante la menstruación" produce el mayor porcentaje de respuestas erradas, y "relaciones sexuales durante la lactancia" fue la situación que ofreció mayores diferencias considerando los grupos etarios.

La mitad de los adultos considera que pueden ser métodos anticonceptivos que la mujer orine antes y después de la relación sexual, que se ponga de pie luego del coito o que la penetración se realice sin preservativo de modo de retirar el pene antes de la eyaculación. Estos datos, sumados a la no concepción durante la lactancia o la menstruación podrían estar indicando un elevado porcentaje de personas que los consideran formas de anticoncepción.

En relación con los porcentajes globales de acierto, no se observan diferencias notables entre ambos grupos etarios, o entre mujeres y varones, ya sea separados por grupos o considerando los de ambos grupos en conjunto.

Los varones adultos son los que más aciertos muestran, mientras que los varones jóvenes obtienen globalmente los porcentajes más bajos. 
Meinardi, E. et al.

\section{Reflexiones finales}

En este apartado final nos gustaría explicitar algunas reflexiones que surgen del conjunto de acciones que realizamos.

Una manera de superar las situaciones desfavorables que expresan las estadísticas en Argentina en relación con la Educación para la Salud Sexual es llevando a cabo acciones concretas en las aulas. Nuestro proyecto se propuso explorar un terreno que prácticamente no tiene antecedentes en Argentina, tanto en la formación del profesorado de biología como en la de estudiantes de nivel medio, jóvenes y adultos. Atravesamos las dificultades propias de un campo nuevo para la investigación didáctica. Sin embargo, estamos convencidos de la necesidad de nuestra tarea. Son muchos los profesores y profesoras que están dispuestos a encarar estos temas desde una perspectiva distinta a la tradicional, es decir, revisando los múltiples factores involucrados en la Educación para la Salud Sexual.

La Educación para la Salud Sexual está conformada por una multiplicidad de enfoques disciplinares que superan ampliamente la mirada biológica. Sería deseable incorporar en el currículo de la formación del profesor de biología diversas perspectivas de las ciencias sociales respecto de la sexualidad (antropológica, legal, de género etc.). Cuando los docentes en servicio toman conciencia de la falta de formación en estos importantes aspectos suelen dejar de lado su enseñanza. De este proyecto surge la elaboración de materiales didácticos que sirven, a su vez, de orientación a otros profesores interesados en intervenir en sus aulas. La articulación de la investigación didáctica con la formación inicial y continuada del profesorado transforma los problemas del aula en problemas de investigación sobre los que se ensayan intervenciones fundamentadas. Este aspecto del proyecto apunta a generar una didáctica que tenga incidencia en el aula, de modo de lograr trascender el mero discurso.

El relevamiento sobre las concepciones de los estudiantes puso de manifiesto cómo algunas de ellas crean obstáculos con los que tropieza la enseñanza, generando la posibilidad de que los profesores modifiquen sus prácticas a fin de superar dichos obstáculos. Por otro lado, prácticamente no existe la formación docente para la educación de poblaciones en riesgo, por lo que se constituye en un gran desafío contextualizar las estrategias de enseñanza que diseñan los profesores y profesoras que participan de este proyecto.

Los análisis realizados sobre los resultados de esta investigación reafirman que se hace imprescindible una intervención seria, responsable y sostenida con los alumnos de escuela media. No es posible, ni éticamente aceptable esconderse tras los porcentajes que parecen no ser tan altos en términos de respuestas erróneas. En estas subyacen no sólo conceptos erróneos respecto de la longevidad de una célula sexual o la efectividad de un método sino que suponen una concepción determinada de qué es la sexualidad y de cómo ejercerla.

Asumimos entonces el desafío de analizar e investigar qué prácticas, materiales e intervenciones didácticas son las que prometen mejores resultados, es decir, que estén dirigidos a la toma de decisiones responsables y comprendidas, en las que los conocimientos conceptuales sean realmente significativos y no meramente etiquetas.

En este sentido comprendemos que la investigación didáctica debe redundar no sólo en la reflexión respecto de la necesidad de instalar la Educación para la Salud Sexual como temática en la formación de profesores, definir sus alcances y objetivos, sino también el generar materiales didácticos que lleguen a las aulas. $\mathrm{Al}$ principio puede ocurrir que sean estos los 
Educación para la Salud Sexual en la formación...

únicos disponibles generados desde esta nueva perspectiva, pero esperamos que los profesores sean cada vez más autónomos y diseñen a su tiempo sus propios materiales, y que multipliquen y socialicen sus experiencias.

\section{Agradecimientos}

A la Agencia Nacional de Promoción de las Investigaciones Científicas y Tecnológicas de la Argentina, PICT 18-26106, por la financiación del Proyecto en el cual se enmarca este trabajo.

\section{Referencias}

AROMI, A. et al. Saber hacer y tropiezos en el campo educativo sobre la cuestión sexual. Grupo de Investigación sobre Psicoanálisis y Pedagogía. In: ENCUENTRO

INTERNACIONAL DEL CAMPO FREUDIANO, 12., 2002, París. Anais... París, 2002.

Disponível em: <http://www.scb-icf.net/nodus/>. Acesso em: 20 ago. 2008.

BARCELOS, N. S.; ZAIAD, A. G.; SANTOS, C. Educação sexual: relato de uma experiência. Revista Brasileira de Sexualidade Humana, v. 7, ed. esp. 2, p. 150-160, 1996.

BERNARDI, M. A deseducação sexual. São Paulo: Summus, 1985.

BRANDÃO, E. R.; HEILBORN, M. L. Sexualidade e gravidez na adolescência entre jovens de camadas médias do Rio de Janeiro, Brasil. Cadernos de Saúde Pública, v. 22, n. 7, p. 1421-1430, 2006.

BRÊTAS, J. R. S.; SILVA, C. V. Orientação sexual para adolescentes: relato de experiência. Acta Paulista de Enfermagem, v. 18, n. 3, p. 326-333, 2005.

CAAMAÑO CANO, V. M. Educación sexual en el aula: asignaturas pendientes para el profesorado. Novedades Educativas, n. 150, p. 12-15, 2003.

CERUTI BASO, S. Sexualidad y adolescencia: la sexualidad en el contexto de la salud integral de los adolescentes. In: MADDALENO, M. et al. (Eds.). La salud del adolescente y del joven. Washington: OPS, 1995. p. 141-142.

CONDERS. Consorcio Nacional de Derechos Reproductivos y Sexuales. Diagnóstico de la situación de la atención de la salud sexual y reproductiva desde la perspectiva de las/os usuarias/os, 2003. Disponível em: <http://www.conders.org.ar/html/ Frameset.html>. Acesso em: 20 ago 2008.

CROSS, K. P. Adults as learners. San Francisco: Jossey-Bass, 1981.

DAPÍA CONDE, M. D.; MEMBIELA, P.; CID, M. C. Un proyecto curricular de ciencias orientado hacia la Educación para la Salud. Alambique, n. 9, p. 57-62, 1996. 
Meinardi, E. et al.

DENTE, L.; ROGALSKI, R. Hablemos a calzón quitado: la escuela en tiempos de SIDA. Buenos Aires: CTERA, 1999.

GAVIDIA, V. La escuela promotora de salud y sostenibilidad. Didáctica de las Ciencias Experimentales y Sociales, n. 18, p. 65-80, 2004.

.; RODES, M. J. Las actitudes hacia la salud. Alambique, n. 22, p. 87-96, 1999.

.; __ Tratamiento de la Educación para la Salud como materia transversal.

Alambique, n. 9, p. 7-16, 1996.

INDEC. Instituto Nacional de Estadística y Censos, Argentina. Censo Nacional 2001.

Disponível em: <http://www.indec.mecon.ar>. Acesso em: 20 ago. 2008.

LÓPEZ GARCÍA, M. Educación afectivo-sexual. Planteamiento integrado e interdisciplinar. Madrid: Narcea - Ministerio de Educación y Ciencia, 1995.

MANDÚ, E. N.; CORRÊA, A. C. Educação sexual formal na adolescência: contribuições à construção de projetos educativos. Acta Paulista de Enfermagem, v. 13, n. 1, p. 27-37, 2000 .

MARGULIS, M. Juventud, cultura, sexualidad: la dimensión cultural en la afectividad y la sexualidad de los jóvenes de Buenos Aires. Buenos Aires: Biblos, 2003.

MEINARDI, E. Reflexiones sobre la formación inicial de los profesores de biología.

Revista de Educación en Biología, v. 10, n. 2, p. 48-54, 2007.

. Educación para la salud sexual y formación del profesorado: una agenda pendiente en Argentina. Enseñanza de las Ciencias, número extra, 2005. Disponível em: $<$ http://ensciencias.uab.es/webblues/www/congres2005/htm/aa.htm>. Acesso em: 20 ago. 2008 .

.; ADÚRIZ-BRAVO, A.; REVEL-CHION, A. La Educación Ambiental en el aula. Una propuesta para integrar contenidos multidisciplinares a través de la argumentación. Investigación en 1a Escuela, n. 46, p. 93-103, 2002.

OMS - Europa. Health Promotion. A discusión document on the concepts and principles. Copenhagen: OMS, 1985.

SILVA, M. P.; CARVALHO, W. L. P. O desenvolvimento do conhecimento pedagógico do conteúdo de sexualidade na vivência das professoras. Ciência \& Educação, v. 11, n. 1, p. 73-82, 2005.

PEREIRO NÚÑEZ, C.; PIZARRO SÁNCHEZ, J. Comunicación persona a persona. In: AA.VV. Aprendiendo Ciencias en la Enseñanza Secundaria. Santiago de Compostela: Universidad de Santiago de Compostela, 1997. p. 397-432.

RAMOS, R. Representaciones sociales de docentes de una escuela media acerca de la sexualidad y de la educación sexual: estudio de caso. 2007. Tesis (Maestría en Salud Mental) - Facultad de Trabajo Social, Universidad Nacional de Entre Ríos, Argentina, 2007. 
Educación para la Salud Sexual en la formación...

REVEL CHION, A.; BONAN, L.; MEINARDI, E. Salud sexual y salud reproductiva: necesidad de una separación. Enseñanza de las Ciencias, n. extra, 2005. Disponível em: <http://ensciencias.uab.es/webblues/www/congres2005/htm/aa.htm>. Acesso em: 20 ago. 2008.

ROKEACH, M. Enciclopedia Internacional de las Ciencias Sociales. Madrid: Aguilar, 1974.

SÁNCHEZ, C.; SANTOS, H. La educación sexual en la escuela. Buenos Aires: Grupo Editor Latinoamericano, 1986.

SANTOS, H. Algunas consideraciones pedagógicas sobre la educación sexual. In: CLEMENT, A. (Org.). Educación sexual en la escuela: perspectivas y reflexiones. Buenos Aires: Ministerio de Educación - GCBA, 2006. p. 5-22.

SANTOS, H. Salud reproductiva. In: TALLERES DE ANÁLISIS DE LA PERTINENCIA SOCIAL DE LAS INVESTIGACIONES DE LA UBA EN EL ÁREA-PROBLEMA: Salud Reproductiva, 1., 2003, Buenos Aires. Programa... Buenos Aires: Secretaría de Ciencia y Técnica, 2003. Disponível em: <http://www.rec.uba.ar/ progevext.htm>. Acesso em: 20 ago. 2008

SCHOLER, A. M. Sexuality in the science classroom: one teacher's methods in a college biology course. Sex Education: Sexuality, Society and Learning, v. 1, n. 2, p. 75-86, 2002.

SHULMAN, L. Those who understand: knowledge growth in teaching. Educational Researcher, v. 2, n. 15, p. 4-14, 1986.

TEIXEIRA, A. M. F. B. et al. Adolescentes e uso de preservativos: as escolhas dos jovens de três capitais brasileiras na iniciação e na última relação sexual. Cadernos de Saúde Pública, v. 22, n. 7, p. 1385-1396, 2006. 